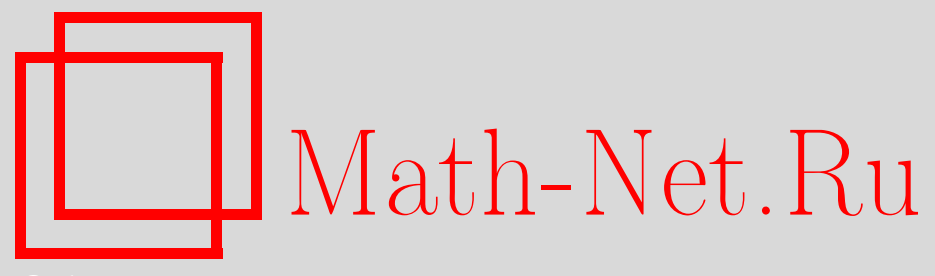

Н. В. Ильченко, Критерий полноты для неоднородных функций с задержками, Дискрет. матем., 1996, том 8, выпуск 1, 86-98

DOI: https://doi.org/10.4213/dm510

Использование Общероссийского математического портала Math-Net.Ru подразумевает, что вы прочитали и согласны с пользовательским соглашением http: //www.mathnet.ru/rus/agreement

Параметры загрузки:

IP : 54.197 .217 .227

26 апреля 2023 г., 15:00:14 
УДК 519.7

\title{
Критерий полноты для неоднородных функций с задержками
}

(C) 1996 г. Н. В. Ильченко

\begin{abstract}
Рассмотрена функциональная система неоднородных функций $f:\{0,1\}^{n} \rightarrow C, C \in\{\{0,1\},\{0,3\}\}$ с задержками $t$ из $\mathbf{N}_{0}=\{0,1,2, \ldots\}$, т. е. пар $(f, t)$ с операциями синхронной суперпозиции. Для нее в терминах предполных классов решена задача описания всех ф-полных множеств, т.е. таких, из әлементов которых с помощью указанных операций можно получить пару $(f, t)$ для любой функции $f$. Из этого описания вытекает алгоритмическая разрешимость задачи об ф-полноте.
\end{abstract}

\section{1. Введение}

Рассматривается функциональная система $\bar{P}_{\Sigma}$ пар $(f, t)$ с операциями синхронной суперпозиции (с-суперпозиции), где $t \in\{0,1,2, \ldots\}$ и

$$
f:\{0,1\}^{n} \rightarrow\{a, b\}, \quad\{a, b\} \in\{\{0,1\},\{0,3\}\} .
$$

Пара $(f, t)$ называется неоднородной функцией $f$ с задержкой $t$. Система $\bar{P}_{\Sigma}$ является ближайшим обобщением булевых функций с задержками с операциями с-суперпозиции. Основным результатом работы является установление критерия, позволяющего выяснить для любой системы указанных пар возможность из нее с помощью операций с-суперпозиции получить любую из указанных неоднородных функций хоть с какой-нибудь задержкой - свойство функциональной полноты (ф-полноты). Из критерия вытекает алгоритмическая разрешимость задачи об ф-полноте. Необходимые понятия и результаты заимствуются из [1, 2] с упрощенными обозначениями.

\section{2. Основные понятия и результаты}

Пусть $E_{2}=\{0,1\}, D=\{0,3\}, V \in\left\{E_{2}, D\right\}, \mathbf{N}=\{1,2, \ldots\}, \mathbf{N}_{0}=\mathbf{N} \cup\{0\}$ и $U=\left\{u_{1}, u_{2}, \ldots, u_{n}, \ldots\right\}-$ множество переменных со значениями из $E_{2}$, переменные из $U$ будем обозначать метасимволами $x_{i}, i=1,2, \ldots$ Обозначим через $P_{V}$ класс всех функций $f\left(x_{1}, \ldots, x_{n}\right)$ со значениями из $V$, а через $P_{\Sigma}$ класс $P_{E_{2}} \cup P_{D}$. Для $P_{E_{2}}$ далее используем принятое обозначение $P_{2}$, а его элементы назовем булевыми функциями. Если $K \subseteq P_{\Sigma}$, полагаем $K_{V}=K \cap P_{V}$.

Пусть $K \subseteq P_{\Sigma}$. Введем операции суперпозиции над $K$ следующим образом. 
(1) Пусть $f\left(x_{1}, \ldots, x_{n}\right) \in K$ и $u_{j_{1}}, u_{j_{2}}, \ldots, u_{j_{n}} \in U$. Тогда $f\left(u_{j_{1}}, u_{j_{2}}, \ldots, u_{j_{n}}\right)$ задает новую функцию из $P_{\Sigma}$, полученную по определению из $f$ с помощью операций суперпозиции (операции переименования переменных).

(2) Пусть $f\left(x_{1}, \ldots, x_{n}\right) \in K$ и $g_{1}, \ldots, g_{n}$ - строка переменных из $U$ или функций из $P_{2}$, которые получены из $K$ с помощью операций суперпозиции. Тогда функция $g$, определяемая выражением $f\left(g_{1}, \ldots, g_{n}\right)$, по определению получена из $f$ и $g_{1}, \ldots, g_{n}$ с помощью операций суперпозиции (подстановка функций и переменных в функцию).

Для $K \subseteq P_{\sigma}$ через $[K]$ обозначим множество всех функций, полученных из $K$ с помощью конечного числа применений операций (1) и (2), и назовем его замыканием множества $K$. Назовем множество $K$ замкнутым, если $[K]=K$, и полным в $P_{\Sigma}$, если $[K]=P_{\Sigma}$. Множество $K$ называется предполным, если $K$ не полно и для любой $f \in P_{\Sigma} \backslash K$

$$
[K \cup\{f\}]=P_{\Sigma}
$$

Введем классы $\bar{P}_{V}=\left\{(f, t): f \in P_{V}, t \in \mathbf{N}_{0}\right\}$ и $\bar{P}_{\Sigma}=\bar{P}_{2} \cup \bar{P}_{D}$. Для обозначения подмножеств из $\bar{P}_{\Sigma}$ будем использовать символ $\widetilde{K}$. Пусть $p \in \mathbf{N}_{0}$, положим

$$
\widetilde{K}^{p}=\{(f, t):(f, p) \in \tilde{K}\}, \quad \tilde{K}^{(1)}=\{f:(f, t) \in \tilde{K}\} .
$$

Пусть $\bar{Q} \in\left\{\bar{P}_{2}, \bar{P}_{\Sigma}\right\}$ и $\widetilde{K} \subseteq \bar{Q}$. Введем понятие операции с-суперпозиции над $\widetilde{K}$ следующим образом.

$\left(1^{\prime}\right)$ Пусть $\left(f\left(x_{1}, \ldots, x_{n}\right), t\right) \in \widetilde{K}$ и $u_{j_{1}}, u_{j_{2}}, \ldots, u_{j_{n}} \in U$. Тогда выражение $\left(f\left(x_{i_{1}}, \ldots, x_{i_{n}}\right), t\right)$ задает пару $(g, t)$ из $\bar{Q}$, в которой $g$ реализуется выражением $f\left(u_{j_{1}}, u_{j_{2}}, \ldots, u_{j_{n}}\right)$. Пара $(g, t)$ по определению получается из $\left(f\left(x_{1}, \ldots, x_{n}\right), t\right)$ с помощью операции с-суперпозиции (с-переименование переменных).

$\left.\left(2^{\prime}\right) 2^{\prime}\right)$ Пусть $\left(f\left(x_{1}, \ldots, x_{n}\right), t\right) \in \widetilde{K}$ и $\left(g_{1}, \tau\right), \ldots,\left(g_{n}, \tau\right)$ - строка пар из $\bar{Q}_{E_{2}}$, которые получены из $\widetilde{K}$ с помощью операций с-суперпозиции, или при $\tau=0$ могут также иметь вид $\left(x_{j}, 0\right)$. Тогда выражение $\left(f\left(g_{1}, \ldots, g_{n}\right), t+\tau\right)$ задает по определению элемент из $\bar{Q}$, полученный из $(f, t)$ и $\left(g_{1}, \tau\right), \ldots,\left(g_{n}, \tau\right)$ с помощью операций с-суперпозиции (с-подстановка пар в пару).

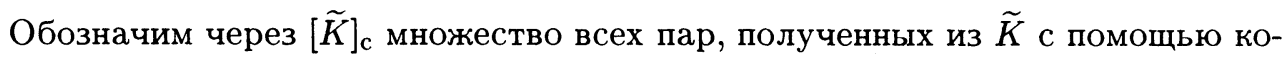
нечного числа применений операций $\left(1^{\prime}\right)$ и $\left(2^{\prime}\right)$, и назовем его с-замыканием множества $\widetilde{K}$.

Назовем множество $\widetilde{K}$ с-замкнутым, если $[\widetilde{K}]_{\mathrm{c}}=\widetilde{K}$. Множество $\widetilde{K}$ называется с-полным в $\bar{Q}$, если $[\widetilde{K}]_{\mathrm{c}}=\bar{Q}$. Множество $\widetilde{K}$ называется с-предполным в $\bar{Q}$, если $\widetilde{K}$ не с-полно, но для любого элемента $(f, t) \in \bar{Q} \backslash \widetilde{K}$

$$
[\widetilde{K} \cup\{(f, t)\}]_{\mathrm{c}}=\bar{Q} \text {. }
$$

Задача о полноте для $\bar{P}_{\Sigma}$ состоит в решении уравнения $[\tilde{K}]_{\mathrm{c}}=\bar{P}_{\Sigma}$ относительно $\widetilde{K}$, т.е. в описании всех с-полных множеств. В [3] она решена путем отыскания всех с-предполных классов в $\bar{P}_{\Sigma}$. 
Для $K \subseteq P_{\Sigma}$ обозначим через $\bar{K}$ множество всех пар из $\bar{P}_{\Sigma}$ вида $(f, t)$, где $f \in K$ и $t \in \mathbf{N}_{0}$.

Множество $\widetilde{K} \subseteq \bar{Q}$ называется ф-полным в $\bar{Q}$, если $[\widetilde{K}]_{\mathbf{c}}^{(1)}=Q$. Пусть $t \in \mathbf{N}_{0}$, $\mathbf{N}^{\prime} \subseteq \mathbf{N}_{0}$. Обозначим через $\widetilde{R}_{t}$ любое множество вида

$$
\bigcup_{j \in \mathbf{N}^{\prime}}\left(f_{j}, k_{j} t\right) \cup\{(x, t)\}
$$

где $k_{j} \in \mathbf{N}$, такое, что $\left[\widetilde{R}_{t}\right]_{\mathrm{c}}^{(1)}=P_{2}$.

Справедливо следующее утверждение.

Теорема 1. Множество $\widetilde{K} \subseteq \bar{P}_{\Sigma}$ является ф-полныц в $\bar{P}_{\Sigma}$ тогда и только тогда, когда для некоторых $t, T \in \mathbf{N}_{0}, \widetilde{R}_{t} u f \in P_{D} \backslash\{0,3\}$

$$
[\widetilde{K}]_{\mathrm{c}} \supseteq \widetilde{R}_{t} \cup\{(f, T)\} .
$$

Следствие 1. Любое ф-полное множество содерэсит конечное ф-полное подмножество.

Пусть $L, S, M, T_{0}, T_{1}$ - соответственно классы линейных, самодвойственных, монотонных, сохраняющих 0 , сохраняющих 1 булевых функций.

Если $f\left(x_{1}, \ldots, x_{n}\right) \in P_{2}$, то при совпадении $f(x, \ldots, x)$ с $x, 1,0$ или $\bar{x}$ назовем $f$ соответственно функцией типа $\langle\alpha\rangle,\langle\beta\rangle,\langle\gamma\rangle,\langle\delta\rangle$, обозначая соответственно классы всех таких функций через $A, B, \Gamma, \Delta$.

Функция $f\left(x_{1}, \ldots, x_{n}\right) \in P_{2}$ называется четной, если $f\left(x_{1}, \ldots, x_{n}\right)=$ $f\left(\bar{x}_{1}, \ldots, \bar{x}_{n}\right)$. Пусть $Y$ - класс всех таких функций.

Пусть $r \in \mathbf{N}, \mathbf{N}_{r}=\{1,2, \ldots, r\}, \mathfrak{M}_{E}=\{3\} \cup P_{2}, 0=\{0\}, 1=\{1\}$.

Введем следующие классы: $\overline{\mathfrak{M}}_{E}, \bar{L}, \bar{S}, \bar{T}_{0}, \bar{T}_{1}, \widetilde{H}=\bar{S}^{0} \cup\left(\bar{Y} \backslash \bar{Y}^{0}\right)$,

$$
\begin{aligned}
\tilde{C}= & \bar{A}^{0} \cup\left(\bar{B} \backslash \bar{B}^{0}\right) \cup\left(\bar{\Gamma} \backslash \bar{\Gamma}^{0}\right), \\
\tilde{E}_{0}= & \bar{A}^{0} \cup \bar{B}^{0} \cup\left(\overline{0} \backslash \overline{0}^{0}\right) \cup \overline{1}, \\
\tilde{E}_{1}= & \bar{A}^{0} \cup \bar{\Gamma}^{0} \cup \overline{0} \cup\left(\overline{1} \backslash \overline{1}^{0}\right), \\
\widetilde{W}_{r}= & \left\{\left(f,(2 t+1) 2^{r}\right): \bar{f} \in M, t \in \mathbf{N}_{0}\right\} \\
& \cup\left\{\left(\varphi,(2 t+1) 2^{s}\right): \varphi \in M, s \in \mathbf{N} \backslash \mathbf{N}_{r}, t \in \mathbf{N}_{0}\right\} \\
& \cup \bar{M}^{0} \cup \overline{1} \cup \overline{0}, \quad r \in \mathbf{N}_{0}, \\
\tilde{Z}_{r}= & \left\{\left(f,(2 t+1) 2^{r}\right): f \in \Delta, t \in \mathbf{N}_{0}\right\} \\
& \cup\left\{\left(\varphi,(2 t+1) 2^{s}\right): \varphi \in A, s \in \mathbf{N} \backslash \mathbf{N}_{r}, t \in \mathbf{N}_{0}\right\} \cup \bar{A}^{0}, \quad r \in \overline{\mathbf{N}}_{0} .
\end{aligned}
$$

Пусть

$$
\mathfrak{L}=\left\{\bar{L}, \bar{S}, \bar{M}, \bar{T}_{0}, \bar{T}_{1}, \widetilde{H}, \tilde{C}, \tilde{E}_{0}, \tilde{E}_{1}\right\} \cup \bigcup_{r \in \mathbf{N}_{0}}\left\{\widetilde{W}_{r}\right\} \cup \bigcup_{r \in \mathbf{N}_{0}}\left\{\tilde{Z}_{r}\right\}
$$

Если $\widetilde{K} \subseteq \bar{P}_{\Sigma}$, то ${ }^{0}[\widetilde{K}]_{\mathrm{c}}=[\widetilde{K}]_{\mathrm{c}} \cap \overline{0}$. 
Теорема 2. Множсетво $\widetilde{K} \subseteq \bar{P}_{\Sigma}$ является ф-полным в $\bar{P}_{\Sigma}$ тогда и только тогда, когда $\widetilde{K}_{B} \nsubseteq \overline{\mathfrak{M}}_{E}$ и для любого $Q$ из $\mathfrak{L}$

$$
\widetilde{K}_{E_{2}} \cup^{0}[\tilde{K}]_{\mathrm{c}} \nsubseteq Q
$$

Теорема 3. Распознавание свойства ф-полноты конечных жножсеств в $\bar{P}_{\Sigma}$ алгоритмически разрешимо.

Множество $\tilde{K}$ называется ф-предполным в $\bar{P}_{\Sigma}$, если оно не является фполным, но для всякой пары $(f, t),(f, t) \in \bar{P}_{\Sigma} \backslash \widetilde{K}$, множество $\widetilde{K} \cup\{(f, t)\}$ ф-полно в $\bar{P}_{\Sigma}$.

Обозначим через $\Gamma^{*}$ множество всех функций $f\left(x_{1}, \ldots, x_{n}\right) \in P_{D}$ таких, что $f(0, \ldots, 0)=f(1, \ldots, 1)=0$.

Функция $f\left(x_{1}, \ldots, x_{n}\right)$ называется $D$-самодвойственной, если на всякой паре наборов $\vec{\alpha}=\left(\alpha_{1}, \ldots, \alpha_{n}\right)$ и $\vec{\beta}=\left(\bar{\alpha}_{1}, \ldots, \bar{\alpha}_{n}\right)$, называемых противоположными,

$$
(f(\vec{\alpha}), f(\vec{\beta})) \neq(0,0) \text {. }
$$

Обозначим через $P_{D, S}$ класс всех не $D$-самодвойственных функций.

Рассмотрим множества

$$
\begin{gathered}
\bar{T}_{0}^{*}=\bar{T}_{0} \cup \bar{P}_{D}, \quad \bar{M}^{*}=\bar{M} \cup \bar{P}_{D}, \quad \bar{L}^{*}=\bar{L} \cup \bar{P}_{D}, \quad \tilde{E}_{1}^{*}=\tilde{E}_{1} \cup \bar{P}_{D}, \\
\bar{T}_{1}^{*}=\bar{T}_{1} \cup\left(\bar{P}_{D} \backslash \bar{\Gamma}^{*}\right), \quad \bar{S}^{*}=\bar{S} \cup\left(\bar{P}_{D} \backslash \bar{P}_{D, S}\right), \\
\tilde{C}^{*}=\tilde{C} \cup\left(\bar{P}_{D} \backslash \bar{\Gamma}^{* 0}\right), \quad \tilde{E}_{0}^{*}=\tilde{E}_{0} \cup\left(\bar{P}_{D} \backslash \Gamma^{* 0}\right), \quad \widetilde{H}^{*}=\tilde{H} \cup\left(\bar{P}_{D} \backslash \bar{P}_{D, S}^{0}\right), \\
\widetilde{W}_{r}^{*}=\widetilde{W}_{r} \cup \bar{P}_{D}, \quad \tilde{Z}_{r}^{*}=\tilde{Z}_{r} \cup\left(\bar{P}_{D} \backslash \Gamma^{*}\right), \quad r \in \mathbf{N}_{0} .
\end{gathered}
$$

Обозначим

$$
\widetilde{\mathfrak{L}}=\left\{\bar{T}_{0}^{*}, \bar{M}^{*}, \bar{L}^{*}, \tilde{E}_{0}^{*}, \bar{T}_{1}^{*}, \bar{S}^{*}, \tilde{C}^{*}, \tilde{E}_{0}^{*}, \tilde{H}^{*}\right\} \cup \bigcup_{r \in \mathbf{N}_{0}}\left\{\widetilde{W}_{r}^{*}\right\} \cup \bigcup_{r \in \mathbf{N}_{0}}\left\{\tilde{Z}_{r}^{*}\right\}
$$

Теорема 4. Множсество всех ф-предполных классов в $\bar{P}_{\Sigma}$ совпадает с $\widetilde{\mathfrak{L}} \cup\left\{\overline{\mathfrak{M}}_{E}\right\}$; $\widetilde{K} \subseteq \bar{P}_{\Sigma}$ является ф-полным тогда и только тогда, когда $\widetilde{K} \nsubseteq Q^{*}$ для любого $Q^{*}$ uз $\widetilde{\mathfrak{L}} \cup\left\{\overline{\mathfrak{M}}_{E}\right\}$.

\section{3. Доказательство теоремы 1}

В [1] доказаны следующие леммы.

Лемма 1. Множество $\widetilde{K} \subseteq \bar{P}_{2}$ является ф-полным тогда и только тогда, когда $[\widetilde{K}]_{\mathrm{c}} \supseteq \widetilde{R}_{t}$ для некоторого $t \in \mathbf{N}_{0}$.

Обозначим через $X_{t}$ множество вида $\{(x, t),(\bar{x}, t),(1, t),(0, t)\}$, где $t \in \mathbf{N}_{0}$.

Лемма 2. Если $\widetilde{K} \subseteq \bar{P}_{2}$ конечно, ф-полно $u[\widetilde{K}]_{\mathrm{c}} \supseteq\{(x, t),(\bar{x}, t)\}$ для некоторого $t \in \mathbf{N}$, то найдется такое $\mathbf{t}^{\prime} \in \mathbf{N}_{0}$, что $[\tilde{K}]_{\mathrm{c}} \supseteq X_{t^{\prime}}$. 
Доказательство теоремы 1. Докажем необходимость. Покажем, что если $\widetilde{K}$ фполно, то $\widetilde{K} \supseteq \widetilde{R}_{t} \cup\{(f, T)\}$. Поскольку $\widetilde{K}$ ф-полно, то $\left(\left([\widetilde{K}]_{\mathrm{c}}\right)_{E_{2}}\right)^{(1)}=P_{2}$. Следовательно, по лемме $1 \widetilde{K} \supseteq \widetilde{R}_{t}$ при некотором $t \in \mathbf{N}_{0}$.

Из ф-полноты $\widetilde{K}$ следует, что существует $t^{\prime} \in \mathbf{N}_{0}$ такое, что $\left(f, t^{\prime}\right) \in\left([\widetilde{K}]_{\mathrm{c}}\right)_{D}$ для некоторой функции $f \in P_{D} \backslash\{0,3\}$.

Установим достаточность. Пусть $[\widetilde{K}]_{\mathrm{c}} \supseteq \widetilde{R}_{t} \cup\{(f, T)\}$. Покажем, что $\widetilde{K} \phi-$ полно. Для этого с учетом ф-полноты $\widetilde{R}_{t}$ в $\bar{P}_{2}$ достаточно установить, что для любой функции $g \in P_{D}$ найдется $t_{g}$, такое, что $\left(g, t_{g}\right) \in[\widetilde{K}]_{\mathrm{c}}$. Нетрудно видеть, что если $(h, \tau) \in\left[\widetilde{R}_{t}\right]_{\mathrm{c}}$, то $\tau=a t$. Поэтому с учетом ф-полноты $\widetilde{R}_{t}$ в $\bar{P}_{2}$ при некотором $b \geqslant 0$ имеем $(\bar{x}, b t)$. Таким образом, $\left[\widetilde{R}_{t}\right]_{\mathrm{c}} \supseteq\{(x, t),(\bar{x}, b t)\}$. Отсюда следует, что $\left[\widetilde{R}_{t}\right]_{\mathrm{c}} \supseteq\{(x, c t),(\bar{x}, c t)\}$ при некотором $c \geqslant 0$. По лемме 2 заключаем, что $\left[\widetilde{R}_{t}\right]_{\mathrm{c}} \supseteq X_{t_{1}}$ для некоторого $X_{t_{1}}$.

Рассмотрим $\left(f\left(x_{1}, \ldots, x_{n}\right), T\right)$. Для $f$ найдутся два набора $\vec{\alpha}=\left(\alpha_{1}, \ldots, \alpha_{n}\right)$ и $\vec{\beta}=\left(\beta_{1}, \ldots, \beta_{n}\right)$ такие, что $f(\vec{\alpha}) \neq f(\vec{\beta})$. Подставим в пару $(f, t)$ вместо $x_{i}$ пару $\left(0, t_{1}\right)$, если $\alpha_{i}=\beta_{i}=0$, пару $\left(1, t_{1}\right)$, если $\alpha_{i}=\beta_{i}=1$, пару $\left(x, t_{1}\right)$, если $\alpha_{i}=1$ и $\beta_{i}=0$, пару $\left(\bar{x}, t_{1}\right)$, если $\alpha_{i}=0, \beta_{i}=1, i=1, \ldots, n$.

В результате получим $\left(e(x), T+t_{1}\right)$, причем $e(0) \neq e(1)$. Поскольку $(\bar{x}, t) \in[\widetilde{K}]$, можно считать, что $e(0)=0$ и $e(1)=3$. Пусть $g\left(x_{1}, \ldots, x_{m}\right)$ - произвольная функция из $P_{D}$. Выберем в $\left([\widetilde{K}]_{\mathrm{c}}\right)_{E_{2}}$ пару $\left(\lambda\left(x_{1}, \ldots, x_{m}\right), T^{\prime}\right)$ такую, что $\lambda$ и $g$ равны нулю на одних и тех же наборах. Нетрудно видеть; что, подставляя пару $\left(\lambda, T^{\prime}\right)$ в $\left(e, T+t_{1}\right)$, получаем, что $\left(g, T^{\prime}+t_{1}+T\right) \in[\widetilde{K}]_{\mathrm{c}}$. Теорема доказана.

Интересно отметить, что уточнение теоремы с требованием кратности $T$ в паре $(f, T)$ числу $t$, оказывается, вообще говоря, неверным. Установим это.

Рассмотрим множество $\widetilde{K}=\left\{(x, 2),\left(\bar{x}_{1} \vee \bar{x}_{2}, 2\right),(f, 3)\right\}$, где $f \in P_{D} \backslash\{0,3\}$, а также $\widetilde{K}_{a}=\left\{(x, 2),\left(\bar{x}_{1} \vee \bar{x}_{2}, 2\right)\right\} \cup \overline{0} \cup \overline{1}$ и $\widetilde{K}_{b}=\widetilde{K}_{a} \cup\{(h, 3)\}$, где $h \in P_{D} \backslash\{0,3\}$.

Предложение 1. $E c л u(g, l) \in\left[\widetilde{K}_{a}\right]_{c}$, то при $g \notin\{0,1\}$ число l четно.

Доказательство. Установим утверждение индукцией по числу $l$. Пусть $(g, l) \in$ $\left[\widetilde{K}_{a}\right]_{\mathrm{c}}$ и $l \leqslant 2$, тогда нетрудно убедиться, что $(g, l) \in\left\{(x, 2),\left(\bar{x}_{1} \vee \bar{x}_{2}, 2\right),(0,0),(0,1)\right.$, $(0,2),(1,0),(1,1),(1,2)\}$, т. е. если $g \notin\{0,1\}$, то $l$ четно.

Пусть теперь $n>2$. Предположим, что наше утверждение верно для $l \leqslant n$. Покажем, что оно верно и для $l \leqslant n+1$. Пусть $l \leqslant n+1$ и $(g, l)$ есть результат с-подстановки пар $\left(g_{1}, \tau\right), \ldots,\left(g_{m}, \tau\right)$ в пару $(f, p)$. В этом случае, если $p \leqslant 1$ или $\tau \leqslant 1$, то $g \in\{0,1\}$. Пусть $p>1$ и $\tau \geqslant 1$. Тогда, если $p=2 k+1$ или $\tau=2 r+1$, то по предположению индукции $f \in\{0,1\}$ или $g_{i} \in\{0,1\}$ для каждого $i \in\{1, \ldots, n\}$, следовательно, $g \in\{0,1\}$. Если же $p=2 k$ и $\tau=2 r$, то $l=2(k+r)$ и при $g \in P_{2} \backslash\{0,1\}$ наше утверждение верно. Предложение установлено.

Предложение 2. Ecли $(g, l) \in\left[\tilde{K}_{b}\right]_{c}$, то при $g \notin\{0,3\}$ число l нечетно.

Доказательство. Пусть $(g, l) \in\left[K_{b}\right]_{\mathrm{c}}$ и $g \in P_{D}$. Можно считать, что пара $(g, l)$ получена с-подстановкой пар $\left(g_{1}, \tau\right), \ldots,\left(g_{m}, \tau\right)$ в пару $(h, 3)$, где $h \in P_{D} \backslash\{0,3\}$ и $i \in\{1, \ldots, m\}$. Тогда $g_{i} \in\{0,1\}$, отсюда $g \in\{0,3\}$. 
Далее, по предложению 1 , если $g_{i} \notin\{0,1\}$ при некотором $i$, то $\tau=2 k$. В этом случае результат с-подстановки дает пару $\left(h\left(g_{1}, \ldots, g_{m}\right), 3+2 k\right)=(g, 1+2(k+1))$, где $g \in P_{D}$. Таким образом, в паре $(g, l) \in\left[\widetilde{K}_{b}\right]_{\text {с }}$ при условии, что $g \in P_{D} \backslash\{0,3\}$, имеем $l=1+2 p$. Предложение установлено.

Предложение 3. Условие $[\widetilde{K}]_{\mathrm{c}} \supseteq \widetilde{R}_{t} \cup\{(f, k t)\}$, әде $f \in P_{D} \backslash\{0,3\}$, не является необходимым для ф-полноты $\widetilde{K}$.

Доказательство. Пусть $\widetilde{K}=\left\{(x, 2),\left(\bar{x}_{1} \vee \bar{x}_{2}, 2\right),(h, 3)\right\}$, где $h \in P_{D} \backslash\{0,3\}$. По

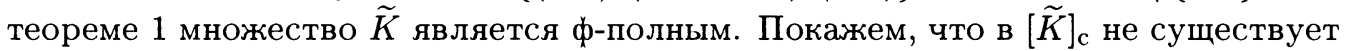
ф-полной системы вида $\widetilde{R}_{t} \cup\{(f, l t)\}$, где $f \in P_{D} \backslash\{0,3\}$.

Если $(g, t) \in[\widetilde{K}]_{\mathrm{c}} \cap \bar{P}_{2}$, то $(g, t) \in\left[\widetilde{K}_{a}\right]_{\mathrm{c}}$, и по предложению 1 при $g \notin\{0,1\}$ имеем $t=2 k$.

Если $(u, t) \in[\tilde{K}]_{\mathrm{c}} \cap \bar{P}_{D}$, то $(u, t) \in\left[\widetilde{K}_{b}\right]_{\mathrm{c}}$, и по предложению 2 при $u \notin\{0,1\}$ имеем $t=2 l+1$.

Таким образом, если $\widetilde{R}_{t} \subseteq[\tilde{K}]_{\mathrm{c}}$, то пары из $[\tilde{K}]_{\mathrm{c}} \cap \bar{P}_{2}$ имеют четную задержку, а пары из $\bar{P}_{D} \cap[\widetilde{K}]_{\mathrm{c}}$ нечетную. Предложение установлено.

\section{4. Доказательство теоремы 2}

В [2] доказано следующее утверждение.

Теорема 5. Множсество $\widetilde{K} \subseteq \bar{P}_{2}$ является ф-полным тогда и только тогда, когда $\widetilde{K} \nsubseteq Q$ для любого $Q \in \mathfrak{L}$.

Предложение 4. Для $\widetilde{K} \subseteq \bar{P}_{\Sigma}$ следующие условия әквивалентны:

(1) $\left([\tilde{K}]_{\mathrm{c}}^{(1)}\right) \supseteq P_{2}$,

(2) $\left(\widetilde{K}_{E_{2}} \cup^{0}[\tilde{K}]_{\mathrm{c}}\right)_{\mathrm{c}}^{(1)}=P_{2}$,

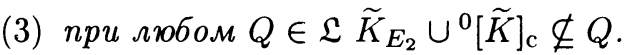

Доказательство теоремы 2. Докажем достаточность. Пусть выполнены условия (а) и (b). Покажем, что $\widetilde{K}$ ф-полно. Из (а) следует, что в $\widetilde{K}$ найдется такая пара $(f, t)$, что $f \in P_{D}$ и $f \notin\{0,3\}$. Из (b) с учетом предложения 4 и леммы 1 получаем, что $[\widetilde{K}]_{\mathrm{c}} \supseteq \widetilde{R}_{t}$ при некотором $t$, откуда по теореме 1 следует ф-полнота множества $\widetilde{K}$.

Докажем необходимость. Пусть $\widetilde{K}$ ф-полно. Тогда по теореме $1[\tilde{K}]_{\mathrm{c}} \ni(f, T)$, где $f \in P_{D} \backslash\{0,3\}$ и $[\widetilde{K}]_{\mathrm{c}} \supseteq \widetilde{R}_{t}$, но $\left[\widetilde{R}_{t}\right]_{\mathrm{c}}^{(1)}=P_{2}$ и поэтому условия (а) и (b) теоремы 2 выполнены. Теорема 2 доказана. 


\section{5. Доказательство теоремы 3}

Установим ряд вспомогательных утверждений.

Лемма 3. Для конечных множсеств $\widetilde{K} \subseteq \bar{P}_{\Sigma}$ свойство $\left\{\right.$ входит ли $(0,0)$ в $\left.[\tilde{K}]_{\mathrm{c}}\right\}$ алгоритмически разрешимо.

Доказательство. Рассмотрим два случая, $\tilde{K}^{0} \subseteq \bar{P}_{2}$ и $\widetilde{K}^{0} \nsubseteq \bar{P}_{2}$. В первом случае, очевидно, включения $(0,0) \in\left[\widetilde{K}^{0}\right]_{\text {с }}$ и $0 \in\left[\widetilde{K}^{0}\right]_{\text {c }}^{(1)}$ эквивалентны. Последнее включение, как известно (см. [4]) алгоритмически разрешимо.

Во втором случае пусть $\widetilde{K}^{0}=\widetilde{K}_{E_{2}}^{0} \cup \widetilde{K}_{D}^{0}, \widetilde{K}_{D}^{0}=\left\{\left(f_{1}, 0\right), \ldots,\left(f_{s}, 0\right)\right\}$ и $n$ - наибольшее число переменных у функций из $\left(\widetilde{K}_{D}^{0}\right)^{(1)}$. Проверка свойства $(0,0) \in\left[\tilde{K}_{E_{2}}^{0}\right]_{\text {с }}$ рассмотрена в первом случае. Пусть $0 \notin\left[\tilde{K}_{E_{2}}^{0}\right]_{\mathbf{c}}^{(1)}$. Заметим, что для любой пары $(f(x), 0)$, где $f(x) \in\{x, \bar{x}, 1\}$, включение $(f(x), 0) \in\left[K_{E_{2}}^{0}\right]_{\mathrm{c}}$, как установлено в [4], алгоритмически разрешимо. Поэтому можно считать, что множество $\left[\widetilde{K}_{E_{2}}^{0}\right]_{\mathrm{c}}^{(1)} \cap\{x, \bar{x}, 1\}$ нам известно. Покажем, как алгоритмически определить вхождение $(0,0) \in\left[\tilde{K}^{0}\right]_{\mathrm{c}}$. С этой целью рассмотрим произвольную пару $\left.f_{i}\left(g_{i_{1}}, \ldots, g_{i_{n_{i}}}\right), 0\right)$, получающуюся из $\left(f_{i}\left(x_{i_{1}}, \ldots, x_{i_{n_{i}}}\right), 0 \in \widetilde{K}_{D}^{0}\right.$ подстановкой в нее пар $\left.g_{i_{j}}(x), 0\right) \in\left[\tilde{K}_{E_{2}}^{0}\right]_{c}$, где $j=1, \ldots, n_{i}, i=1, \ldots, s_{i}$. Таких формально различимых пар не более, чем $s 3^{n}$. При этом, если $(0,0) \in\left[K^{0}\right]_{\mathrm{c}}$, то среди указанных пар обязательно найдется совпадающая с парой $(0,0)$. Таким образом, включение $(0,0) \in\left[\widetilde{K}^{0}\right]_{\text {с }}$ сводится к нахождению пары $(0,0)$ среди построенных пар $\left(f_{i}\left(g_{1}, \ldots, g_{n}\right), 0\right)$, что алгоритмически проверяемо. Лемма доказана.

Лемма 4. Если $\widetilde{K} \subseteq \bar{P}_{\Sigma} u(0,0) \in[\widetilde{K}]_{\mathrm{c}}$, то $\widetilde{K}$ ф-полно тогда и только тогда, когда $\widetilde{K}_{D} \nsubseteq \overline{\mathfrak{M}}_{E}$ u $\widetilde{K}_{E_{2}} \nsubseteq Q$ для любого

$$
Q \in\left\{\bar{M}, \bar{L}, \bar{T}_{0}, \widetilde{E}_{1}\right\} \cup \bigcup_{r \in \mathbf{N}_{0}}\left\{W_{r}\right\}
$$

Доказательство. Необходимость условия $\widetilde{K}_{D} \nsubseteq \overline{\mathfrak{M}}_{E}$ очевидна. Необходимость остальных условий вытекает из того, что множества $\bar{M}, \bar{L}, \bar{T}_{0}, \widetilde{E}_{1}$ и $\widetilde{W}_{r}$ при любом $r$ являются с-замкнутыми, не ф-полными и содержат все пары вида $(0, t)$, $t \geqslant 0$.

Установим достаточность. Пусть $\widetilde{K}_{E_{2}} \cup\{(0,0)\} \nsubseteq Q$ для всех $Q$. Так как $(0,0) \notin Q^{\prime}$ для всех $Q^{\prime} \in \mathfrak{L}$, то $\widetilde{K}_{E_{2}} \cup\{(0,0)\} \nsubseteq Q^{\prime \prime}$ для всех $Q^{\prime \prime} \in \mathfrak{L}$. Отсюда, замечая, что $\widetilde{K}_{D} \nsubseteq \overline{\mathfrak{M}}_{E}$, с учетом теоремы 2 получаем, что $\widetilde{K}$ ф-полно. Лемма доказана.

Следствие 2. При $(0,0) \in[\widetilde{K}]_{\mathrm{c}}$ свойство $\widetilde{K}$ быть ф-полным алгоритмически разрешимо.

Типом $\tau(K)$ множества $K \subseteq P_{2}$ назовем множество всех типов функций из $K$, а основанием $K$ множество всех одноместных функций из $K$. Если $K$ замкнуто, то, очевидно, его тип совпадает с типом основания. 
Лемма 5. Если $\widetilde{K} \subseteq \bar{P}_{\Sigma}, \widetilde{K}$ конечно $и(0,0) \notin[\widetilde{K}]_{c}$, то сөойство \{существует такое $t \in \mathbf{N}$, что $\left.(0, t) \in\left[\widetilde{K}_{E_{2}}\right]_{\mathrm{c}}\right\}$ алгоритмически разрешимо.

Доказательство. Если $\widetilde{K}_{E_{2}} \subseteq \bar{P}_{2}^{0}$, то утверждение очевидно. Пусть $\widetilde{K}_{E_{2}} \nsubseteq \bar{P}_{2}^{0}$. Это означает, что в $\widetilde{K}_{E_{2}}$ имеется пара $\left(f, t^{\prime}\right)$ такая, что $t^{\prime}>0$. Если $\tau\left(K_{E_{2}}\right) \ni\langle\gamma\rangle$, то в $\left[\widetilde{K}_{E_{2}}\right]_{\mathrm{c}}$ имеется, очевидно, пара $\left(0, t^{\prime \prime}\right)$. Подставляя в нее пару $\left(f, t^{\prime}\right)$, получим, что $\left(0, t^{\prime}+t^{\prime \prime}\right) \in\left[\widetilde{K}_{E_{2}}\right]_{\mathrm{c}}$, где $t^{\prime}+t^{\prime \prime}>0$. Если $\tau\left(K_{E_{2}}\right)\langle\gamma\rangle$, то $\tau\left(K_{E_{2}}\right) \in$ $\{\langle\alpha\rangle,\langle\beta\rangle,\langle\alpha, \beta\rangle,\langle\alpha, \delta\rangle\}$, откуда очевидно, что $(0, t) \notin\left[\tilde{K}_{E_{2}}\right]_{\mathrm{c}}$ при любом $t$. Заметим, что условия проверки вхождения типа $\langle\gamma\rangle$ в $\tau\left(K_{E_{2}}\right)$ и получения $\left(0, t^{\prime}+t^{\prime \prime}\right)$ алгоритмически проверяемы. Лемма доказана.

Как установлено в [1], имеет место следующее утверждение.

Предложение 5. Для любого $r \in \mathrm{N}_{0}$

$$
\{(x, t),(\bar{x}, t)\} \nsubseteq \widetilde{Z}_{r}
$$

Пусть $f$ зависит от $x_{1}, \ldots, x_{n}$. Обозначим через $f^{x}$ функцию, задаваемую формулой $f(x, \ldots, x)$.

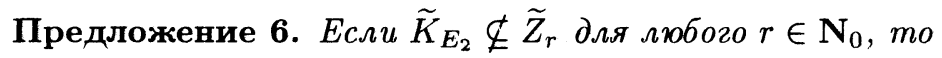

$$
\left[\widetilde{K}_{E_{2}}\right]_{\mathrm{c}} \supseteq\{(x, t),(\bar{x}, t)\} .
$$

Доказательство. Рассмотрим все пары $(f, t)$ из $\tilde{K}_{E_{2}}$ такие, что $\tau(f)=\langle\delta\rangle$. Их множество $\widetilde{K}^{\prime}$ можно записать так:

$$
\widetilde{K}^{\prime}=\left\{\left(f_{i},\left(2 t_{i}+1\right) 2^{r_{i}}\right): f_{i} \in \Delta, i=1,2, \ldots, l\right\},
$$

где $r_{i} \in \mathrm{N}_{0}$ для всех $i$. Здесь либо все $r_{i}$ равны между собой, либо это не так.

Во втором случае можно считать, что $r_{1}<r_{2}$. Рассмотрим пары $\left(f_{1}^{x},(2 t+1) 2^{r_{1}}\right)$ и $\left(f_{2}^{x},(2 t+1) 2^{r_{2}}\right)$. Ясно, что $f_{i}^{x}=\bar{x}$ при $i=1,2$. Подставляя в себя первую пару $\left(2 t_{1}+1\right) 2^{r_{2}-r_{1}}$ раз, а вторую в себя $\left(2 t_{1}+1\right)$ раз, получим, соответственно, пары

$$
\left(x,\left(2 t_{1}+1\right)\left(2 t_{2}+1\right) 2^{r_{2}}\right),\left(\bar{x},\left(2 t_{1}+1\right)\left(2 t_{2}+1\right) 2^{r_{2}}\right) .
$$

В первом случае выделяем в $\widetilde{K}_{E_{2}}$ множество $\widetilde{K}^{\prime \prime}$ всех пар $\left(g, t^{\prime}\right)$, в которых $\tau(g)=\langle\alpha\rangle$. Их можно записать в виде

$$
\left\{\left(g_{j},\left(2 t_{j}^{\prime}+1\right) 2^{r_{j}}\right): g_{j} \in A, j=1,2, \ldots, m\right\} .
$$

Рассмотрим три подслучая.

1. Существует такое $j_{0}$, что $r_{j_{0}}^{\prime}=r_{1}$. Рассмотрим пары

$$
\left(f_{1}^{x},\left(2 t_{1}+1\right) 2^{r_{1}}\right),\left(g_{j_{0}}^{x},\left(2 t_{j_{0}}^{\prime}+1\right) 2^{r_{j}}\right) .
$$

Ясно, что $f_{1}^{x}=\bar{x}$ и $g_{j_{0}}^{x}=x$. Подставляя первую пару в себя $\left(2 t_{j_{0}}+1\right)$, а вторую $\left(2 t_{1}+1\right)$ раз, получаем, соответственно, пары

$$
\left(\bar{x},\left(2 t_{1}+1\right)\left(2 t_{j_{0}}+1\right) 2^{r_{1}}\right),\left(x,\left(2 t_{j_{0}}+1\right)\left(2 t_{1}+1\right) 2^{r_{1}}\right) .
$$


2. Существует такое $j_{0}$, что $r_{j_{0}}^{\prime}<r_{1}$. Рассмотрим пары

$$
\left(f_{1}^{x},\left(2 t_{1}+1\right) 2^{r_{1}}\right),\left(g_{j_{0}}^{x},\left(2 t_{j_{0}}^{\prime}+1\right) 2^{r_{j_{0}}^{\prime}}\right)
$$

Ясно, что $f_{1}^{x}=\bar{x}, g_{j_{0}}^{x}=x$. Подставляя первую пару в себя $\left(2 t_{j_{0}}+1\right)$ раз, а вторую $\left(2 t_{1}+1\right) 2^{r_{1}-r_{j_{0}}}$ раз, получаем, соответственно, пары

$$
\left(\bar{x},\left(2 t_{1}+1\right)\left(2 t_{j_{0}}+1\right) 2^{r_{1}}\right),\left(x,\left(2 t_{1}+1\right)\left(2 t_{j_{0}}+1\right) 2^{r_{1}}\right) .
$$

3. Всегда $r_{j}^{\prime}>r_{1}$. Тогда, очевидно, $\widetilde{K}_{E_{2}} \subseteq Z_{r_{1}}$, что противоречит утверждению. Значит, этого случая быть не может. Утверждение доказано.

Лемма 6. Для конечных множсеств $\widetilde{K} \subseteq \bar{P}_{\Sigma} \operatorname{maxux,~ито~}(0,0) \notin[\widetilde{K}]_{\mathrm{c}} u(0, t) \notin$ $\left[\widetilde{K}_{E_{2}}\right]_{\mathrm{c}}$ nри любых $t$, свойство $\left\{\right.$ существует такое $t^{\prime}>0$, ито $\left.\left(0, t^{\prime}\right) \in[\widetilde{K}]_{\mathrm{c}}\right\}$ алгоритмически разрешимо.

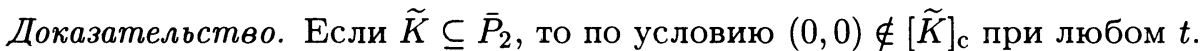

Пусть теперь $\widetilde{K} \nsubseteq \bar{P}_{2}$, т. е. $\widetilde{K} \neq \varnothing$. Возможны два случая, $\widetilde{K}_{E_{2}}=\varnothing$ и $\widetilde{K}_{E_{2}} \neq \varnothing$.

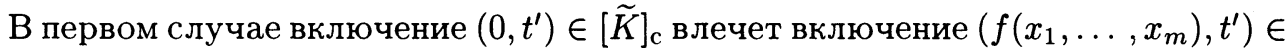

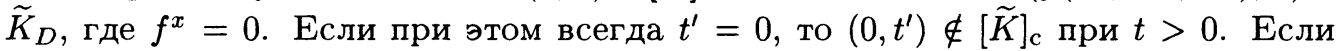
найдется $t^{\prime}>0$, то, так как $\left(f^{x}, t^{\prime}\right)=\left(0, t^{\prime}\right)$, имеем $\left(0, t^{\prime}\right) \in[\widetilde{K}]_{\mathrm{c}}$ и $t^{\prime}>0$.

Оба свойства устанавливаются в силу конечности $\widetilde{K}$ перебором.

Во втором случае будем иметь $\rho=\tau\left(K_{E_{2}}\right) \in\{\langle\alpha\rangle,\langle\beta\rangle,\langle\alpha, \beta\rangle,\langle\alpha, \delta\rangle\}$ и без ограничения общности считаем, что $(x, 0) \in \widetilde{K}_{E_{2}}$, поэтому для $\rho$ значение $\langle\beta\rangle$ далее исключаем.

Если $(0, t) \in[\widetilde{K}]_{c}$, то для некоторых пар $\left(h\left(x_{1}, \ldots, x_{m}\right), t_{1}\right)$ из $\widetilde{K}_{D}$ и $\left(g_{1}, t_{2}\right), \ldots$, $\left(g_{m}, t_{2}\right)$ из $\left[\widetilde{K}_{E_{2}}\right]_{c}$ справедливо равенство

$$
\left(h\left(g_{1}, \ldots, g_{m}\right), t_{1}+t_{2}\right)=(0, t),
$$

а, значит, $h\left(g_{1}^{x}, \ldots, g_{m}^{x}\right)=0$. Рассмотрим следующие случаи.

1. $\rho=\langle\alpha\rangle$. Тогда, поскольку в этом случае $g_{i}^{x}=x$ для всех $i$, имеем $h\left(g_{1}^{x}, \ldots, g_{m}^{x}\right)=h(x, \ldots, x)=0$. Если $t_{1}=0$, то при $\widetilde{K}_{E_{2}} \subseteq \bar{P}_{2}^{0}$ и $t>0$, очевидно, $(0, t) \notin[\widetilde{K}]_{\text {c }}$ п при $\widetilde{K}_{E_{2}} \nsubseteq \bar{P}_{2}^{0}$ найдется пара $\left(g, t^{\prime}\right)$, в которой $t^{\prime}>0$.

Поскольку $\left(g^{x}, t^{\prime}\right)=\left(x, t^{\prime}\right)$, то $\left(x, t^{\prime}\right) \in[\widetilde{K}]_{\mathrm{c}}$, а поэтому, делая с-подстановку пары $\left(x, t^{\prime}\right)$ в $\left(h^{x}, t_{1}+t^{\prime}\right)$, получаем, что $\left(h^{x}, t_{1}+t^{\prime}\right) \in[\widetilde{K}]_{c}$, где $t_{1}+t^{\prime}>0$.

2. $\rho=\langle\alpha, \beta\rangle$. Тогда, поскольку $g_{i}^{x} \in\{1, x\}$ для всех $i$, можно считать, что $h\left(g_{1}^{x}, \ldots, g_{m}^{x}\right)=h(1, \ldots, 1, x, \ldots, x)=0$, а тем самым $h(1, \ldots, 1)=0$.

Если $t_{1}>0$, то, делая с-подстановку произвольной пары $\left(g^{x}, t_{g}\right)$ из $\widetilde{K}_{E_{2}}$ такой, что $\tau(g)=\langle\beta\rangle$, в $\left(h^{x}, t_{1}\right)$, получим, что $\left(h^{x}\left(g^{x}\right), t_{1}+t_{g}\right)=\left(0, t_{1}+t_{g}\right)$, а тем самым

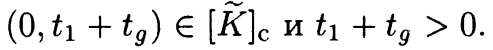

Если во всяком представлении (1) $t_{1}=0$, то при $\widetilde{K}_{E_{2}} \subseteq \bar{P}_{2}^{0}$, заключаем, что

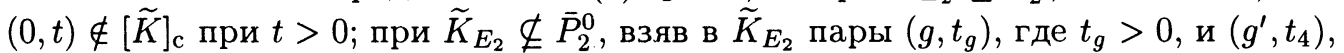
где $\tau\left(g^{\prime}\right)=\langle\beta\rangle$, и, делая с-подстановку $\left(g^{x}, t_{g}\right)$ в $\left(g^{\prime x}, t_{4}\right)$, получаем пару $\left(1, t_{g}+t_{4}\right)$. Сделав с-подстановку этой пары в $\left(h^{x}, t_{1}\right)$, получим $(0, t)$, где $t=t_{1}+t_{g}+t_{4}>0$. 
3. $\rho=\langle\alpha, \beta\rangle$. Тогда, поскольку $g_{i}^{x} \in\{x, \bar{x}\}$ для всех $i$, можно считать, что $h\left(g_{1}^{x}, \ldots, g_{m}^{x}\right)=h(x, \ldots, x, \bar{x}, \ldots, \bar{x})=0$. Здесь или хотя бы одна из групп переменных $x$ и $\bar{x}$ пустая, или это не так. В первом случае рассмотрим пару $\left(h^{x}, t_{1}\right)$. Ясно, что $\left(h^{x}, t_{1}\right)=\left(0, t_{1}\right)$, а, значит, при $t_{1}>0$ имеем $\left(0, t_{1}\right) \in[\widetilde{K}]_{\mathrm{c}}$. Если $t_{1}=0$ во всяком представлении (1), то заключаем, что при $\widetilde{K}_{E_{2}} \subseteq \bar{P}_{2}^{0}$ и $t>0$ справедливо соотношение $(0, t) \notin[\tilde{K}]_{c} ;$ при $\widetilde{K}_{E_{2}} \nsubseteq \bar{P}_{2}^{0}$ берем $\left(g, t_{g}\right)$ из $\widetilde{K}_{E_{2}}$ такую, что $t_{g}>0$. Делаем с-подстановку $\left(g^{x}, t_{g}\right)$ в $\left(h^{x}, t_{1}\right)$, получим пару $\left(0, t_{1}+t_{g}\right)$, где $t_{1}+t_{g}>0$.

Во втором случае в предположении, что первый случай не реализуется при любом представлении (1), в силу предложения (6), можем установить, имеются

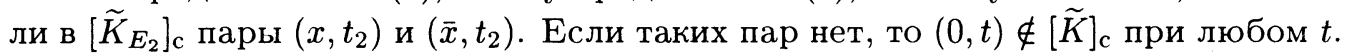
Если такие пары нашлись, то сделаем с-подстановку пары $\left(x, t_{2}\right)$ в $\left(h, t_{1}\right)$ вместо $x_{i}$, если в выражении $h(x, \ldots, x, \bar{x}, \ldots, \bar{x})$ на $i$-м месте стоит $x$, и пары $\left(\bar{x}, t_{2}\right)$, если там стоит $\bar{x}$. В результате получим $\left(0, t_{1}+t_{2}\right)$. Если $\widetilde{K}_{E_{2}} \subseteq \bar{P}_{2}^{0}$ и $t_{1}=0$ для всех пар $\left(h_{1}, t_{1}\right)$, то при $t>0$ имеем $(0, t) \notin[\tilde{K}]_{\mathrm{c}}$. В противном случае с учетом того, что при $\widetilde{K}_{E_{2}} \nsubseteq \bar{P}_{2}^{0}$ можно, очевидно, считать, что $t>0$, либо за счет выбора $t_{1}$ или $t_{2}$, заключаем, что $t_{1}+t_{2}>0$ в паре $\left(0, t_{1}+t_{2}\right)$.

Поскольку все используемые свойства в разобранных случаях устанавливаются перебором $\widetilde{K}$, заключаем, что утверждение леммы справедливо.

Из теоремы 2 и теоремы 1 вытекает справедливость следующего утверждения.

Лемма 7. Если $(0, t) \in[\widetilde{K}]_{\mathrm{c}}$ при некотором $t>0$, то $\widetilde{K}$ ф-полно тогда и только mогда, когда $\widetilde{K}_{D} \nsubseteq \overline{\mathfrak{M}}_{E}$ u $\widetilde{K}_{E_{2}} \nsubseteq Q$ для любого

$$
Q \in\left\{\bar{L}, \bar{M}, \bar{T}_{0}, \widetilde{E}_{0}, \widetilde{H}\right\} \cup \bigcup_{r \in \mathrm{N}_{0}}\left\{\widetilde{W}_{r}\right\}
$$

В работе [2] установлено следующее предложение.

Теорема 6. Существует алгоритм, которьй для любого конечного $\widetilde{K} \subseteq \bar{P}_{2}$ устанавливает, верно ли свойство $\widetilde{K} \nsubseteq Q$ при любом $Q \in \mathfrak{L}$.

Доказательство теоремы 3. Опишем основные шаги алгоритма распознавания ф-полноты для конечного $\widetilde{K} \subseteq \bar{P}_{\Sigma}$.

Шаг 1. Проверка условия $(0,0) \in[\widetilde{K}]_{c}$. По лемме 3 это свойство алгорит.мически разрешимо. Если $(0,0) \in[\tilde{K}]_{c}$, то в силу следствия 2 заключаем, что имеется алгоритм проверки ф-полноты множества $\widetilde{K}$. Если установлено, что $(0,0) \notin[\tilde{K}]_{\mathrm{c}}$, то переходим к шагу 2 .

Шаг 2. Проверяем, существует ли такое $t \in \mathbf{N}$, что $(0, t) \in[\tilde{K}]_{\mathbf{c}}$. Сначала проверим включение $(0, t) \in\left[\widetilde{K}_{E_{2}}\right]_{\mathrm{c}}$. По лемме 5 это свойство проверяется алгоритмически. Если $(0, t) \notin\left[\widetilde{K}_{E_{2}}\right]_{c}$, то с помощью леммы 6 устанавливается вклю-

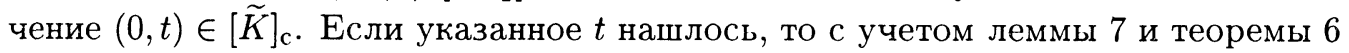
заключаем, что имеется алгоритм, устанавливающий является ли $\widetilde{K} ф$-полным.

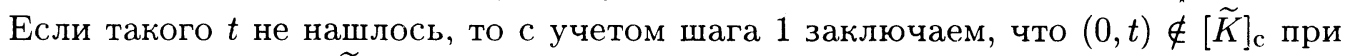
любом $t$ и, значит, $\widetilde{K}$ не является ф-полным. Теорема 3 доказана. 


\section{6. Доказательство теоремы 4}

Обозначим произвольный класс из $\mathfrak{L}$ через $Q$, а соответствующий ему класс из $\widetilde{\mathfrak{L}}$ через $Q^{*}$.

Лемма 8. Классы $\bar{T}_{0}^{*}, \bar{M}^{*}, \bar{L}^{*}, \widetilde{E}_{1}^{*}, \widetilde{W}_{r}^{*}$ nрu $r=0,1,2, \ldots$, суть $\phi$-предполные в $\bar{P}_{\Sigma}$.

Доказательство. Пусть

$$
Q^{*} \in\left\{\bar{T}_{0}^{*}, \bar{M}^{*}, \bar{L}^{*}, \widetilde{E}_{1}^{*}\right\} \cup \bigcup_{r \in \mathbf{N}_{0}}\left\{\widetilde{W}_{r}^{*}\right\}
$$

Покажем, что $Q^{*}$ с-замкнуто, $Q^{*(1)}$ отлично от $P_{\Sigma}$ и $Q^{*}$ ф-полно при добавлении любой пары из $\widetilde{P}_{\Sigma} \backslash Q^{*}$.

Докажем с-замкнутость. По определению $Q^{*}=Q \cup \bar{P}_{D}$, где $[Q]_{\mathrm{c}}=Q,\left[\bar{P}_{D}\right]_{\mathrm{c}}=$ $\bar{P}_{D}$, и, поскольку $Q \cap \bar{P}_{D}=\overline{0}$, то $\left[Q^{*}\right]_{\mathrm{c}}=\left[Q \cup \bar{P}_{D}\right]_{\mathrm{c}}=[Q]_{\mathrm{c}} \cup\left[\bar{P}_{D}\right]_{\mathrm{c}}=Q \cup \bar{P}_{D}$, т. е. $Q^{*}$ замкнуто.

Поскольку $Q^{(1)} \neq P_{2}$ (см. [1]), то $Q^{*(1)} \neq P_{\Sigma}$. Докажем, что $\left[Q^{*} \cup\{(f, t)\}\right]_{\mathrm{c}}^{(1)}=P_{\Sigma}$ при $(f, t) \in \bar{P}_{\Sigma} \backslash Q^{*}$. Ясно, что $\bar{P}_{\Sigma} \backslash Q^{*}=\bar{P}_{2} \backslash Q$. Поэтому $(f, t) \in \bar{P}_{2} \backslash Q$. Поскольку $Q$ ф-предполон в $\bar{P}_{2}$ (см. [1]), то $\left[Q^{*} \cup\{(f, t)\}\right]_{\mathrm{c}} \supseteq[Q \cup\{(f, t)\}]_{\mathrm{c}},[Q \cup\{(f, t)\}]_{\mathrm{c}}^{(1)}=P_{2}$, и так как $Q^{*(1)} \supseteq P_{D}$, то $\left[Q^{*} \cup\{(f, t)\}\right]_{\mathrm{c}}^{(1)} \supseteq\left(P_{2} \cup P_{D}\right)=P_{\Sigma}$. Лемма доказана.

Лемма 9. Классы $\bar{T}_{1}^{*}, \bar{S}^{*}, \widetilde{Z}_{r}^{*}$ при $r=0,1,2, \ldots$, суть ф-предполные в $\bar{P}_{\Sigma}$.

Доказательство. Пусть

$$
Q^{*} \in\left\{\bar{T}_{1}^{*}, \bar{S}^{*}\right\} \cup \bigcup_{r \in \mathbf{N}_{0}}\left\{\widetilde{Z}_{r}^{*}\right\}
$$

Покажем, что $Q^{*}$ с-замкнуто, $Q^{*(1)}$ отлично от $P_{\Sigma}$ и $Q^{*}$ ф-полно при добавлении любой пары из $\bar{P}_{\Sigma} \backslash Q^{*}$.

Докажем с-замкнутость. По определению $Q^{*}=Q \cup \bar{P}_{D} \backslash R$, где $R=\bar{\Gamma}^{*}$ для $\bar{T}_{1}^{*}$ и $\widetilde{Z}_{r}^{*}$ при $r=0,1,2, \ldots$, и $R=\bar{P}_{D, S}$ для $\bar{S}^{*}$. Рассмотрим $\left[Q^{*}\right]_{\mathrm{c}}=$ - $\left[Q \cup\left(\bar{P}_{D} \backslash R\right)\right]_{\mathrm{c}}$. Поскольку $[Q]_{\mathrm{c}}=Q,\left[\bar{P}_{D} \backslash R\right]_{\mathrm{c}}=\bar{P}_{D} \backslash R$, с-подстановка пар из $\bar{P}_{D} \backslash R$ в $Q$ невозможна (по определению с-подстановки), с-переименование переменных и с-подстановка из $Q$ в $\bar{P}_{D} \backslash R$ не дает пар из $\overline{0}$, так как в $\bar{P}_{D} \backslash R$ нет пар вида $(g, T)$, где $g\left(x_{1}, \ldots, x_{n}\right) \equiv 0$. Таким образом,

$$
\left[Q^{*}\right]_{\mathrm{c}}=\left[Q \cup\left(\bar{P}_{D} \backslash R\right)\right]_{\mathrm{c}}=[Q]_{\mathrm{c}} \cup\left[\bar{P}_{D} \backslash R\right]_{\mathrm{c}}=Q \cup\left(\bar{P}_{D} \backslash R\right)=Q^{*}
$$

Поскольку $(0, t) \notin Q^{*}$, то $Q^{*(1)} \neq P_{\Sigma}$.

Докажем теперь, что $\left[Q^{*} \cup\{(f, t)\}\right]_{\mathrm{c}}^{(1)}=P_{\Sigma}$ при $(f, t) \in \bar{P}_{\Sigma} \backslash Q^{*}$. Пусть $(f, t) \epsilon$ $\bar{P}_{\Sigma} \backslash Q^{*}$, тогда либо $(f, t) \in \bar{P}_{2} \backslash Q$, либо $(f, t)=(0, t)$. Однако, в силу строения $Q$ имеем $(0, t) \in \bar{P}_{2} \backslash Q$. Следовательно, $(f, t) \in \bar{P}_{2} \backslash Q$, и в силу ф-предполноты $Q$ в $\bar{P}_{2}$ (см. [1])

$$
\left[Q^{*} \cup\{(f, t)\}\right]_{\mathrm{c}}^{(1)} \supseteq[Q \cup\{(f, t)\}]_{\mathrm{c}}
$$

и $[Q \cup\{(f, t)\}]_{\mathrm{c}}^{(1)}=P_{2}$. Таким образом,

$$
\left[Q^{*} \cup\{(f, t)\}\right]_{\mathrm{c}}^{(1)}=\left[Q \cup\left(\bar{P}_{D} \backslash R\right) \cup\{(f, t)\}\right]_{\mathrm{c}}^{(1)} \supseteq\left(P_{2} \cup P_{D}\right)=P_{\Sigma} .
$$

Значит, $Q^{*}$ ф-предполное. Лемма доказана. 
Лемма 10. Классы $\widetilde{C}^{*}, \widetilde{E}_{0}^{*}, \widetilde{H}^{*}$ суть ф-предпољные.

Доказательстөо. Пусть $Q^{*} \in\left\{\widetilde{C}^{*}, \widetilde{E}_{0}^{*}, \widetilde{H}^{*}\right\}$. Покажем, что $Q^{*}$ с-замкнуто, $Q^{*(1)}$ отлично от $P_{\Sigma}$ и $Q^{*}$ ф-полно при добавлении любой пары из $\widetilde{P}_{\Sigma} \backslash Q^{*}$.

Докажем с-замкнутость. По определению $Q^{*}=Q \cup \bar{P}_{D} \backslash R^{0}$, где $R^{0}=\bar{\Gamma}^{* 0}$ для $\widetilde{C}^{*}, \widetilde{E}_{0}^{*}$, и $R^{0}=\bar{P}_{D, S}^{0}$ для $\bar{H}^{*}$. Далее, $\left.[Q]_{\mathrm{c}}=Q, \bar{P}_{D} \backslash R^{0}\right]_{\mathrm{c}}=\bar{P}_{D} \backslash R^{0}$. Рассмотрим $\left[Q^{*}\right]_{\mathrm{c}}^{0}$. Ясно, что $\left[Q^{*}\right]_{\mathrm{c}}^{0}=\left[Q^{0} \cup\left(\bar{P}_{D} \backslash R^{0}\right)\right]_{\mathrm{c}}^{0}$; с-подстановка пар из $\left(\bar{P}_{D} \backslash R^{0}\right)^{0}$ в $Q^{0}$ невозможна (по определению с-подстановки), с-переименование переменных и сподстановка из $Q^{0}$ в $\left(\bar{P}_{D} \backslash R^{0}\right)^{0}$ не дает пары вида $(0,0)$, поскольку в $\left(\bar{P}_{D} \backslash R^{0}\right)^{0}$ нет пар вида $(g, 0)$, где $g\left(x_{1}, \ldots, x_{n}\right) \equiv 0$. Таким образом, $\left[Q^{*}\right]_{\mathrm{c}}^{0}=\left[Q^{0}\right]_{\mathrm{c}} \cup\left[\left(\bar{P}_{D} \backslash R^{0}\right)^{0}\right]_{\mathrm{c}}$. Поскольку $\left(Q^{*} \backslash Q^{* 0}\right)_{\mathrm{c}}^{(1)} \supseteq P_{D}$, то с-подстановка пар из $Q$ в $(h, T) \in\left(\bar{P}_{D} \backslash R^{0}\right) \cap \bar{P}_{D}$, где $T>0$ дает пару из $\bar{P}_{D}$ с задержкой, большей нуля. С-подстановка пар из множества $\left\{(h, T): h \in P_{D}, T \in \mathbf{N}\right\}$ возможна в $Q$ при условии, что $h\left(x_{1}, \ldots, x_{n}\right) \equiv$ 0 , и дает пару $\left(0, T^{\prime}\right)$, где $T^{\prime}>0$. Однако, по определению $Q$, при некотором $T^{\prime}>0$, имеем $\left(0, T^{\prime}\right) \in Q$. Таким образом, $\left[Q^{*}\right]_{\mathrm{c}}=Q^{*}$.

Поскольку $Q^{(1)} \neq P_{2}$, то $Q^{*(1)} \neq P_{\Sigma}$.

Докажем, что $\left[Q^{*} \cup\{(f, t)\}\right]_{\mathrm{c}}^{(1)}=P_{\Sigma}$ при $(f, t) \in \bar{P}_{\Sigma} \backslash Q^{*}$. Поскольку справедливо включение $(f, t) \in \bar{P}_{\Sigma} \backslash Q^{*}$, то либо $(f, t) \in \bar{P}_{2} \backslash Q$, либо $(f, t)=(0,0)$. Однако, в силу строения $Q$ имеем $(0,0) \in \bar{P}_{2} \backslash Q$. Следовательно, в силу ф-предполноты $Q$ в $\bar{P}_{2}$ (см. [1])

$$
\left[Q^{*} \cup\{(f, t)\}\right]_{\mathrm{c}}^{(1)}=\left[Q \cup\{(f, t)\} \cup\left(\bar{P}_{D} \backslash R^{0}\right)\right]_{\mathrm{c}}^{(1)} \supseteq\left(P_{2} \cup P_{D}\right)=P_{\Sigma}
$$

Лемма доказана.

Лемма 11. Класс $\overline{\mathfrak{M}}_{E}$ является $\phi$-предполным в $\bar{P}_{\Sigma}$.

Доказательство. Покажем, что $\overline{\mathfrak{M}}_{E}$ с-замкнуто, $\mathfrak{M}_{E}$ отлично от $P_{\Sigma}$ и $\overline{\mathfrak{M}}_{E}$ ф-полно при добавлении любой пары из $\bar{P}_{\Sigma} \backslash \overline{\mathfrak{M}}_{E}$.

С-замкнутость $\overline{\mathfrak{M}}_{E}$ следует из того, что $\left[\bar{P}_{2}\right]_{\mathrm{c}}=\bar{P}_{2}$ и

$$
\left[\left\{(3, t): t \in \mathbf{N}_{0}\right\}\right]_{\mathrm{c}}=\left\{(3, t), t \in \mathbf{N}_{0}\right\} \text {. }
$$

С-подстановка пар из $\bar{P}_{2}$ в $\left\{(3, t), t \in \mathbf{N}_{0}\right\}$ дает множество $\left\{(3, t): t \in \mathbf{N}_{0}\right\}$, пары из которого, в свою очередь с-подставлять нельзя по определению ссуперпозиции.

Поскольку $(g, T) \in \bar{P}_{\Sigma}$, где $g \in P_{D}$ и $g \notin\{0,3\}$, и пара $(g, T) \notin \overline{\mathfrak{M}}_{E}$, имеем $\mathfrak{M}_{E} \neq P_{\Sigma}$.

$\Phi$-полнота $\overline{\mathfrak{M}}_{E}$ при добавлении любой пары $(f, T) \in \bar{P}_{\Sigma} \backslash \overline{\mathfrak{M}}_{E}$ следует из теоремы 2.

Лемма 11 доказана.

Доказательство теоремы 4. Пусть $\widetilde{K}$ ф-полно. Следовательно, в силу ф-предполноты оно не является подмножеством ни одного из классов системы $\widetilde{\mathfrak{L}} \cup\left\{\overline{\mathfrak{M}}_{E}\right\}$.

Пусть $\widetilde{K}$ не лежит ни в одном из ф-предполных классов системы $\widetilde{\mathfrak{L}} \cup\left\{\overline{\mathfrak{M}}_{E}\right\}$.

Пусть $Q^{*} \in \tilde{\mathfrak{L}}$ и $\left(f_{Q^{*}}, t\right) \notin Q^{*}$. Рассмотрим множество $F$ пар $(f, t)$ таких, что $\left(f_{Q^{*}}, t\right) \notin Q^{*}$ для всех $Q^{*} \in \tilde{\mathfrak{L}}$, которое в силу ф-предполноты классов $Q^{*}$ 
из $\tilde{\mathfrak{L}}$ не пусто. В силу строения $F$ для всякого $Q^{*} \in \tilde{\mathfrak{L}}$ имеем $\left(f_{Q^{*}}, t\right) \in \bar{P}_{2} \backslash$ $Q$. Таким образом, $F$ не является подмножеством ни одного из ф-предполных в $\bar{P}_{2}$ множеств системы $\mathfrak{L}$, и, следовательно, по критерию ф-полноты в $\bar{P}_{2}$ [2], множество $F$ является ф-полным в $\bar{P}_{2}$, а значит, $[F]_{\mathrm{c}} \supseteq \widetilde{R}_{t}$.

Рассмотрим пару $(f, t) \notin \overline{\mathfrak{M}}_{E}$. Ясно, что для $Q^{\prime} \in \tilde{\mathfrak{L}} \cup\left\{\overline{\mathfrak{M}}_{E}\right\}$ выполнено соотношение $F \cup\{(f, t)\} \notin Q^{\prime}$, и, следовательно, по теореме 2 с учетом включения $\left[\widetilde{R}_{t} \cup\{(f, t)\}\right]_{\mathrm{c}} \supseteq[F \cup\{(f, t)\}]_{\mathrm{c}}$ имеем

$$
F \cup\{(f, t)\}]_{\mathrm{c}}^{(1)} \subseteq\left[\widetilde{R}_{t} \cup\{(f, t)\}\right]_{\mathrm{c}}^{(1)}=P_{\Sigma},
$$

причем $[F \cup\{(f, t)\}]_{c}^{(1)}=P_{\Sigma}$. Таким образом, $\widetilde{K}=F \cup\{(f, t)\}$ ф-полно.

Теорема доказана.

\section{Список литературы}

1. Кудрявцев В. Б. Функциональные системы. Изд-во МГУ, Москва, 1982.

2. Кудрявцев В. Б. Теорема полноты для одного класса автоматов без обратных связей. Проблемы кибернетики (1962) 8, 91-115.

3. Волкова Е. Н., Ильченко Н. В. Критерий полноты для двух систем функций с задержками. В сб.: Логико-алгебрачческие конструкции. Тверь, 1992.

4. Яблонский С. В., Гаврилов П. П., Кудрявцев В. Б. Фұнккии алгебры логики и классы Поста. Наука, Москва, 1966.

5. Яблонский С. В. Вөедение в дискретную математику. Наука, Москва, 1979.

Статья поступила 28.01.93. 